\title{
Waveguide bandpass filters with attenuation of higher-order passbands
}

\author{
Wolfgang Menzel*, Jan Machac** \\ * Microwave Techniques, University of Ulm, D-89069 Ulm, \\ Germany \\ * * Faculty of Electrical Engineering, Czech Technical University, \\ Technicka 2, 10627 Prague 6, Czech Republic
}

\section{Abstract}

A new method is proposed to provide additional attenuation to higher-order passbands of metal waveguide bandpass filters. To this end, shunt waveguide sections with cut-off frequencies are included into the filter structure. Design and results of such filters are presented.

\section{Introduction}

All transmission line filters show a periodic passband/stopband behaviour, either with $n^{*} f_{0}$ or $(2 n-1) * f_{0}$ depending on the use of either $\lambda / 2$ or $\lambda / 4$ line sections. For many applications, higherorder passbands are not acceptable, and great efforts are made to suppress at least part of them employing additional filter elements or resonators, e.g. $/ 1 /$.

Another way to handle these problems could be the inclusion of losses, but only at frequencies above the desired passband. Such a solution becomes relatively easy using metal waveguide circuits with their inherent highpass characteristics. To this end, waveguide elements are added to the filter structure, providing for example lossy shunt impedances to the filter above their cutoff frequency.

Two possible solutions of waveguide bandpass filters of this kind are shown in Fig. 1. In Fig. 1a, the filter resonators are loaded with matched shunt waveguides. Below their cut-off frequency, they provide only some reactive load to the resonators (which can be compensated for), but above that frequency, they provide losses to the filter. However, at the second passband, i.e. when the resonators are about one wavelength long, the electric field in the center of the shunt waveguides becomes very low resulting only in a negiigible attenuation of that passband.

A modified structure is shown in Fig. 1b. In this case, the coupling elements (impedance inverters) between the filter resonators are combined with similar shunt waveguides as in Fig. 1a. Symmetric structures were chosen to avoid the excitation of the $\mathrm{TE}_{20}$-mode in the resonators.

\section{Theory}

For the design of the filter, both basic filter design and characterization of the involved discontinuities had to be included.

The waveguide crossings with the inductive posts in their centers (Fig. 2a) were calculated using a mode matching technique based on 121. Making use of the double symmetry of the structure, only a $90^{\circ}$ bend with different combinations of electric and magnetic walls in the symmetry planes had to be considered (Fig. 2b). In the center region, the superposition of two field setups (Fig. 2c) was chosen according to /2/.

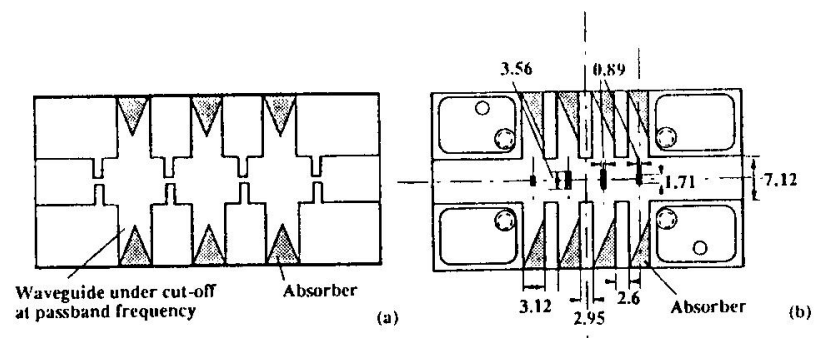

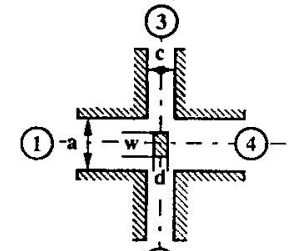

(2) (a)

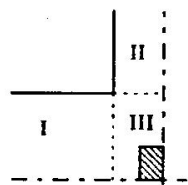

(b)

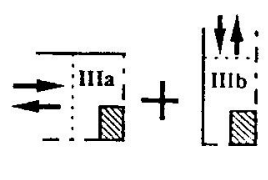

(c)
Fig. 2: Waveguide junction with shunt post (a) and setup for mode matching calculations $\{b, c\}$

The discontinuities within these substructures, once again, were calculated using standard mode matching techniques. A typical result of a waveguide junction as it is used in the filters is shown in Fig. 3.

The filter design itself is based on an equivalent circuit formed by shunt inductances (according to the waveguide junctions) and transmission line resonators. The elements of this equivalent circuit can be easily computed, e.g. according to $/ 1 /$. Following this, the dimensions of the waveguide posts in the junctions were chosen in such a way that the waveguide junctions exhibit the same $\left|S_{41}\right|$ as the shunt inductances of the equivalent circuit. Finally, the resonator lengths were corrected according to the phase angle differences between equivalent inductances and waveguide discontinuities.

\section{Results}

A first filter circuit with three resonators and equal widths of all shunt waveguides was designed for $35 \mathrm{GHz}$ (Chebycheff, $0.1 \mathrm{~dB}$ ripple). Fig. 4 shows theoretical and experimental results in the passband region. For the theoretical curve, the actual dimensions of the filter were taken; due to additional inaccuracies, however, a slight frequency shift between both curves is found. Additionally, the wideband insertion loss is plotted up to $110 \mathrm{GHz}$. At $48 \mathrm{GHz}$, a spurious response around $-30 \mathrm{~dB}$ occurs due to the cut-off frequency of the shunt waveguides. At higher frequencies, measurements became critically, as higher order modes in the Kaband waveguide and resonances affected both calibration and measurement. Therefore, a strong ripple occurred. Nevertheless, a sufficient agreement between theory and experiment can be seen, and the inherent possibilities given by such an arrangement can be recognized already.
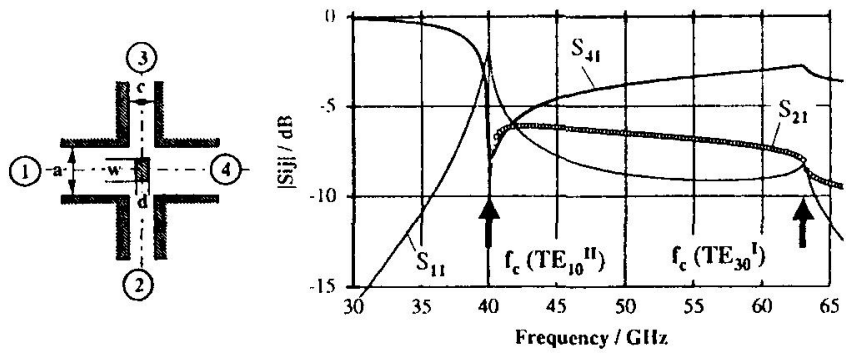

Fig. 3: Waveguide junction and transmission properties $(a=7.12 \mathrm{~mm}, c=3.75 \mathrm{~mm}, w=2.2 \mathrm{~mm}$ $\mathrm{d}=0.88 \mathrm{~mm}$ )

Further improvements are possible, for example, choosing different widths of the shunt waveguides. For the same filter parameters, but with two different widths of the shunt waveguides, a considerable improvement in the $40-50 \mathrm{GHz}$ range was achieved (Fig. 5). For comparison, the response of a comparable filter with shunt posts, but without any shunt waveguide, is shown in this figure, too, showing that the second passband is suppressed by about $30 \mathrm{~dB}$.

Problems with higher passband are even more pronounced in broadband filters. Fig. 6 shows filter responses for three different 
types of filters with $2 \mathrm{GHz}$ bandwidth at $30 \mathrm{GHz}$. Here again, the principle reported in this paper gives an improvement of 18 to 30 $\mathrm{dB}$ in the frequency range from 40 to $65 \mathrm{GHz}$.
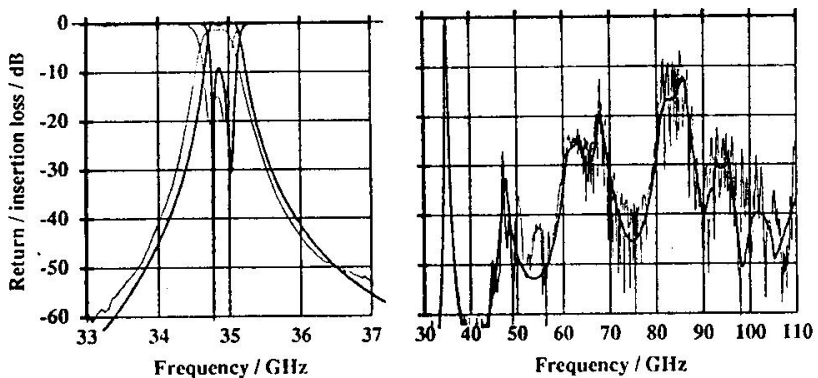

Fig 4: Experimental ( $\rightarrow$ ) and theoretical ( $\longrightarrow$ ) transmission properies of a realized fitter (dimensions see Fig. lb)

\section{Conclusion}

It has been shown that the inclusion of shunt waveguides into bandpass filters can considerably improve the stopband performance in a frequency range where normally higher order passbands occur. This effect should be even more pronounced for filter of higher order, especially when the cut-off frequencies of the shunt waveguides are staggered in a proper way.

\section{References}

/1/ Matthaei, G.; Young, L.; Jones, E.M.T.: Microwave Filters, Impedance Matching Networks, And Coupling Structures. Artech House, 1980, p. $486 \mathrm{ff}$.

/2/ Kühn, E.: A mode-matching method for solving field problems in waveguide and resonator circuits. AEÜ, vol. 27 (1973), 511-518.

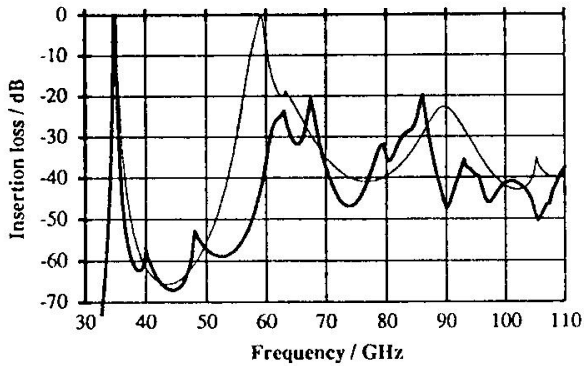

Fig. 5: Theoretical transmission coeflicient of a modified filter $\left(-, c_{1}=3.75 \mathrm{~mm}, c_{2}=3.12 \mathrm{~mm}\right.$ $\left.w_{1}=2.2 \mathrm{~mm}, w_{2}=3.56 \mathrm{~mm}, d_{1}=d_{2}=0.88 \mathrm{~mm}, l_{1}=2.32 \mathrm{~mm}, 1_{2}=2.935 \mathrm{~mm}\right)$ compared to $\mathrm{a}$ standard filter type without shunt waveguides $\left(-, w_{1}=1.4 \mathrm{~mm}, w_{2}=3.2 \mathrm{~nm}, d_{1}=d_{2}=02\right.$ $\mathrm{mm}, 1_{1}=4.92 \mathrm{~mm}, \mathrm{l}_{2}=5.21 \mathrm{~mm}$

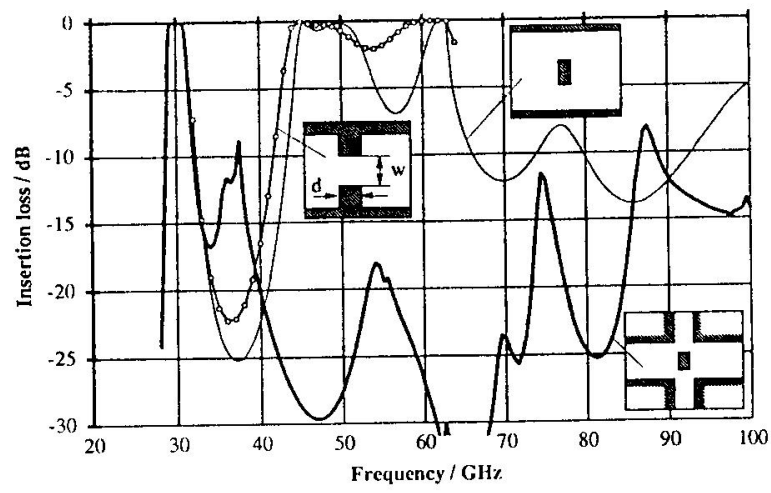

Fig. 6: Transmission coefficient of $2 \mathrm{GHz}$ wide filters with 3 resonators each $(\mathrm{a}=7.12 \mathrm{~mm})$ Filter with inductive irises: $w_{1}=4.5 \mathrm{~mm}, w_{2}=3.5 \mathrm{~mm}, d_{1}=1.16 \mathrm{~mm}, d_{2}=1.12 \mathrm{~mm}$ $l_{1}=4.85 \mathrm{~mm}, l_{2}=5.49 \mathrm{~mm}$

Filter with inductive posts: $w_{1}=0.1 \mathrm{~mm}, w_{2}=1 \mathrm{~mm}, d_{1}=d_{2}=0.2 \mathrm{~mm}, 1_{1}=5.53 \mathrm{mmm}$, $h_{2}=6.03 \mathrm{~mm}$.

Filter with inductive posts and shunt waveguides:
$c_{1}=4.2 \mathrm{~mm}, c_{2}=4 \mathrm{~mm}, w_{1}=0.8 \mathrm{~mm}, w_{2}=1.4 \mathrm{~mm}, d_{1}=0.5 \mathrm{~mm}, d_{2}=1 \mathrm{~mm}, l_{1}=1.87 \mathrm{~mm}$ $c_{1}=4.2 \mathrm{~mm}$
$\mathrm{l}_{2}=2.76 \mathrm{~mm}$ 